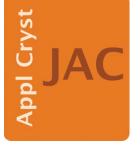

JOURNAL OF APPLIED CRYSTALLOGRAPHY

ISSN 1600-5767

Keywords: book reviews.

Handbook of Industrial Crystallization

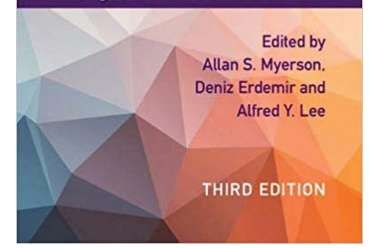

(C) 2020 International Union of Crystallography

\section{Handbook of Industrial Crystallization. Third edition. Edited by Allan S. Myerson, Deniz Erdemir and Alfred Y. Lee. Cambridge University Press, 2019. Pp. 538. Price GBP 145 (hardcover). ISBN 9780521196185.}

\author{
Klaus-Werner Benz* \\ Freiburger Materialforschungszentrum, Freiburg, Germany. *Correspondence e-mail: kw-benz@t-online.de
}

This handbook is the third edition on the topic Industrial Crystallization; the first edition was published by Cambridge University Press in 1993. The book contains 16 chapters (527 pages) written by different authors and covers most basic and applied topics of industrial crystallization. Chapters 1-6 deal with basic and fundamental aspects in the special field of industrial crystallization. Crystal growth means here in particular growth from various types of solutions.

Chapter 1: Solutions and Solution Properties. The first chapter provides a very good survey of important two- and multicomponent solutions related to crystal growth processes. A wide variety of solubility in various solvents as well as large differences of solubility of inorganic components in water is highlighted. Other topics are solubility in mixed solvents, supersaturation and metastability. Fundamental thermal properties affect the energy transfer during the change of temperature in active crystallizers.

Chapter 2: Crystals and Crystal Growth. This contains basic concepts of crystals and a short introduction to the seven crystal systems and Bravais lattices. Mentioned are some defects in perfect crystals like Frenkel and Schottky defects. More space should have been given to the discussion of defects in order to help readers understand the principles of crystal growth phenomena. Defects often play a major part in the quality and size of crystals. A broad intense description of morphology of crystals, including morphological prediction of the expected shape of crystals, is given

Chapter 3: Crystal Nucleation. This chapter is concentrated on crystallization from solutions. A basis for the formation of a nucleus by homogeneous and heterogeneous nucleation is given. Intensively discussed are the origin of secondary nucleation, its nucleation phenomena, nucleation kinetics and control of nucleation.

Chapter 4: The Influence of Impurities and Additives on Crystallization. Strategies to improve the purification and the quality of the resulting crystals are important. The authors describe in detail the influence of foreign species on the growth rate and crystal properties.

Chapter 5: Molecular Modeling Applications in Crystallization. Described are different computational nucleation methods to analyse crystal structures, and to predict morphology and polymorphism. Moreover, these methods are used to solve crystal structures from X-ray powder diffraction data.

Chapter 6: Crystallization Process Analysis by Population Balance Modeling. In order to obtain e.g. an appropriate particle size distribution within the crystallization process, the authors use mathematical models of population balance.

Chapter 7: Selection and Design of Industrial Crystallizers. The authors describe performance criteria in connection with several crystallization methods, which may lead to the design of crystallizers. The configurations of crystallizers strongly influence the product quality. An overview of the main crystallizer types with their characteristics is given.

Chapter 8: Precipitation Processes. The authors describe the complex process of precipitation with very small crystals. The particle concentration is usually between 1011 and 1017 particles per cubic centimetre and the crystal size between 0.1 and $100 \mu \mathrm{m}$. Physical and thermodynamic properties providing the driving force for precipitation are discussed. The kinetics of primary and secondary nucleation play an important role.

Chapter 9: Melt Crystallization. The benefit of melt growth is that heat transfer is the dominating process, whereas in solution growth mass transfer is the most important 
process. The authors describe different types of melt crystallization, including solid-layer crystallization and suspension crystallization. Post-crystallization treatments like sweating and washing are applied to obtain the desired purity of the product. The concepts behind commercial plants are also addressed.

Chapter 10: Crystallizer Mixing. The topic of this chapter is the importance of improving the knowledge of crystallizer flows coupled with heat and mass transport. Crystallizer types are presented and illustrated with the focus on crystallization from solutions in agitated suspensions. Additional computer modelling gives some insight into the existence of turbulent and multiphase flows.

Chapter 11: Monitoring and Advanced Control of Crystallization Processes. This chapter is a review of approaches of crystallization process control and crystallization monitoring. The classification of measurement techniques is specified in sufficient detail. This includes liquid-phase monitoring as well as solid-phase monitoring. Additional modelling and parameter estimation is also discussed.

Chapter 12: Batch Crystallization. Batch crystallization is often used as an open system for manufacturing a variety of crystalline products. The authors describe four types of batch crystallizers (laboratory as well as industrial systems). Batch crystallizers are analysed in detail by developing suitable models and using time-dependent batch conservation equations.

Chapter 13: Crystallization in the Pharmaceutical Industry. The materials of interest have molecular weights between 200 and 700 and are organic compounds. The authors discuss the role of APIs in the pharmaceutical crystal growth processes. Matching the seed characteristics with the cooling profile will ensure controlled crystallization.

Chapter 14: Crystallization of Proteins. This chapter is a review of physical principles that are important for the crystallization of proteins. It is a clearly written article, which covers thermodynamic questions, crystal nucleation with theoretical considerations and experimental data. Described in detail are mechanisms of crystal growth like the generation of steps and kinks and their impact on crystal perfection.

Chapter 15: Crystallization in Foods. The goal of crystallization in food is to generate many crystals with small mean size and narrow distribution. It is described how, in some cases, it is necessary to prevent crystallization in order to achieve an improved product quality.

Chapter 16: Precipitation and Crystallization of Pigments. The authors illustrate the design and scale up of pigment precipitation processes. Practical applications, special aspects and typical process technologies are explained by a few examples.

In its experimental and theoretical aspects, the present handbook is well organized. One of the major strengths of the book is the fact that, despite its wide range of topics, the editors succeeded in combining the different contributions in a very coherent framework. The book represents a good overview of knowledge of the widespread field of industrial crystallization. I recommend the book to scientists and engineers working in this field. 